\title{
Electronic structure and crystal-field states in $\mathrm{V}_{2} \mathrm{O}_{3}{ }^{\mathrm{n}}$
}

\author{
Z. Ropka

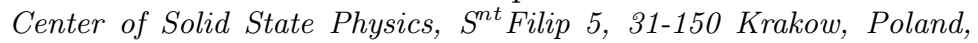 \\ Institute of Physics, Pedagogical University, 30-084 Krakow, Poland \\ R. J. Radwanski

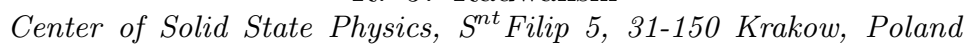 \\ Institute of Physics, Pedagogical University, 30-084 Krakow, Poland
}

\begin{abstract}
We have calculated the electronic structure of $\mathrm{V}_{2} \mathrm{O}_{3}$ associated with the $\mathrm{V}^{3+}$ ions taking into account strong on-site electron correlations and the spin-orbit coupling. Closely lying 9 states of the subterm ${ }^{3} \mathrm{~T}_{1 \mathrm{~g}}$ are a physical reason for exotic phenomena of $\mathrm{V}_{2} \mathrm{O}_{3}$. Electronic structure and magnetism of $\mathrm{V}^{3+}$ ions in the octahedral surroundings are strongly susceptible to lattice distortions and magnetic interactions. Our approach accounts both for the insulating ground state, magnetism, including its orbital contribution, as well as thermodynamical properties.
\end{abstract}

PACS numbers: 71.70.-d, 75.10.Dq

Keywords: $\mathrm{V}_{2} \mathrm{O}_{3}$, crystal field, spin-orbit coupling, strong correlations

$\mathrm{V}_{2} \mathrm{O}_{3}$ attracts a great scientific interest by more than 50 years [1]. Despite of it there is still strong discussion about the description of its properties and its electronic structure. A controversy for $\mathrm{V}_{2} \mathrm{O}_{3}$ starts already with the electronic ground state. Within a localized paradigm there is a long-standing controversy between a $S=1$ model without an orbital degeneracy [2] and the historically first $S=1 / 2$ orbitally degenerate model of Castellani et al. 11. A spin-1 model with three degenerate orbitals was worked out by Di Matteo et al. [3], whereas Refs [4, 5] develop a model, where the fluctuations of $\mathrm{t}_{2 g}$ orbitals and frustrations play prominent role in magnetism of a sister compound $\mathrm{LaVO}_{3}$.

It is agreed that $\mathrm{V}_{2} \mathrm{O}_{3}$ is an insulating antiferromagnet with the Neel temperature of 155-160 K. The basis of all theories is the description of the $\mathrm{V}^{3+}$ ion ( $2 \mathrm{~d}$ electrons) and its electronic structure in the corundum structure, in which the $\mathrm{V}$ ions are placed in a slightly distorted oxygen octahedron.

The aim of this paper is to present a low-energy electronic structure of $\mathrm{V}_{2} \mathrm{O}_{3}$ as originating from the atomiclike electronic structure of the strongly-correlated $3 d^{2}$ electronic systems occurring in the $\mathrm{V}^{3+}$ ions. In our description local atomic-scale effects, the orbital magnetism and the intra-atomic spin-orbit coupling play the fundamentally important role. According to our approach to $3 \mathrm{~d}$ compounds, that seems to be very natural, but is far from being accepted within the present magnetic solid-state community, each $\mathrm{V}$ ion in $\mathrm{V}_{2} \mathrm{O}_{3}$ has two $d$ electrons that form a strongly correlated atomic-like $3 d^{2}$ electron system. These strong correlations among the $3 d$ electrons we account for by two Hund's rules, that yield the ${ }^{3} F$ ground term, Fig. 1a. In the oxygen octahedron surroundings, realized in the corundum structure of

*URL: http://www.css-physics.edu.pl Electronic address: sfradwan@cyf-kr.edu.pl

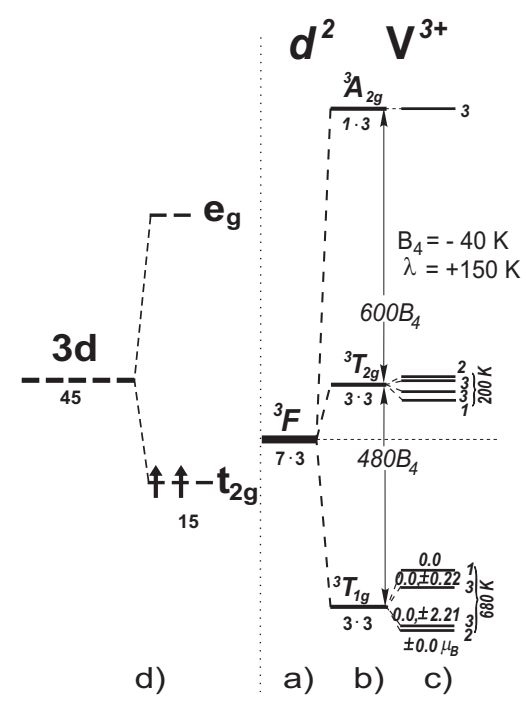

FIG. 1: Lowest part of the electronic structure of $\mathrm{V}_{2} \mathrm{O}_{3}$ associated with the $\mathrm{d}^{2}\left(\mathrm{~V}^{3+}\right)$ ion in the strongly-correlated limit. a) Hund's rules ground term ${ }^{3} F$, b) splitting by the octahedral CEF, c) many-electron CEF states in the octahedral $\mathrm{CEF}$ in the presence of the intra-atomic spin-orbit coupling: $\mathrm{B}_{4}=-40 \mathrm{~K}\left(=10 \mathrm{Dq}=600 \mathrm{~B}_{4}=2.06 \mathrm{eV}\right)$ and the spin-orbit coupling as is observed in the free ion, i.e. $\lambda_{s-o}=+150 \mathrm{~K} . \mathrm{d}$ ) a schematic electronic structure customarily recalled in the current literature - this single-electron electronic structure is, according to us, completely oversimplified.

$\mathrm{V}_{2} \mathrm{O}_{3}$, the ${ }^{3} \mathrm{~F}$ term splits into two orbital triplets ${ }^{3} \mathrm{~T}_{1 g}$, ${ }^{3} \mathrm{~T}_{2 g}$ and an orbital singlet ${ }^{3} A_{2 g}$. In the octahedron of negative oxygen ions the ground subterm is the orbital triplet ${ }^{3} \mathrm{~T}_{1 g}$ as is shown in Fig. 1b. All orbital states are triply spin degenerated. This degeneration is removed by the intra-atomic spin-orbit coupling that is always present in the ion/atom. The effect of the spin-orbit coupling is shown in Fig. 1c. For low-temperature properties the nine states of the ${ }^{3} \mathrm{~T}_{1 g}$ subterm are important. 
Off-octahedral distortions cause further splitting of the shown states - important is that there is no more lowenergy states as only shown. The present calculations have been performed with a realistic octahedral CEF parameter The spin-orbit coupling effect amounts only to $3 \%$ the CEF effect but it has enormous influence on the eigen-functions and low-temperature properties.

The derived many-electron electronic structure of the $\mathrm{d}^{2}$ configuration is completely different from very simplified electronic structure, shown in Fig. 1d, usually presented in the current literature with two parallel spinelectrons in the triply degenerated $t_{2 g}$ orbitals. We do not know, why such an oversimplified scheme is usually shown though the many-electron CEF approach is known already by almost 70 years (it is true that it was rather used not to a solid, but to 3d ions as impurities in Electron Paramagnetic Resonance (EPR) experiments) [6, 7]. A $t_{2 g}^{2}$ state, usually recalled in the literature, is related to the very strong crystal-field limit notation and is 15 fold degenerated. It contains 4 subterms: ${ }^{3} \mathrm{~T}_{1 g},{ }^{1} \mathrm{~T}_{2 g}$, ${ }^{1} \mathrm{E}_{g},{ }^{1} \mathrm{~A}_{1 g}$ also with the lowest ${ }^{3} \mathrm{~T}_{1 g}$ subterm. In our approach to 3d-ion compounds we assume the CEF to be stronger than the spin-orbit coupling, but not so strong to destroy intra-atomic coulombic correlations. Thus we assume an on-site intermediate crystal-field limit, keeping the atomic identity of the involved cation, to be physically adequate for description of 3d-ion compounds.

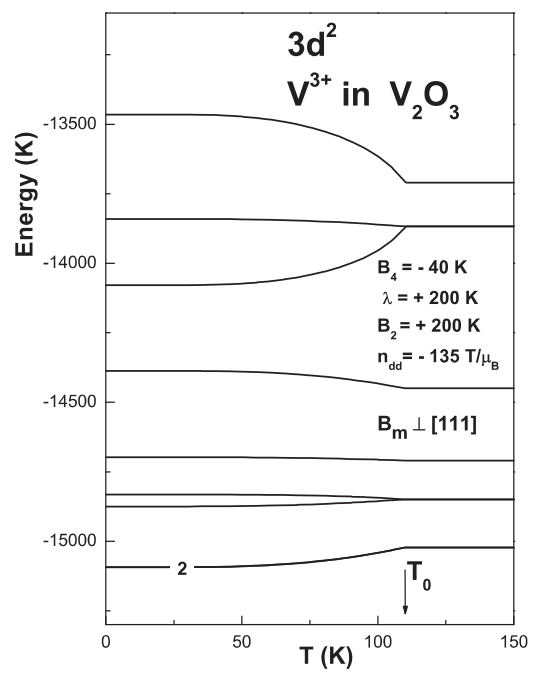

FIG. 2: Modification of the electronic structure (9 lowest states) of the $\mathrm{d}^{2}\left(\mathrm{~V}^{3+}\right)$ ion in a magnetic state. The parameters shown, with the trigonal distortion $\mathrm{B}_{2}=200 \mathrm{~K}$, yield a magnetic moment of $1.19 \mu_{B}$ close to the experimental value of $1.2 \mu_{B}$ and the magnetic ordering temperature of $110 \mathrm{~K}$.
For a magnetic state self-consistent calculations have been performed similarly to those presented in Ref. 8 for $\mathrm{FeBr}_{2}$. A value of $1.19 \mu_{B}$ is in perfect agreement with experimental observation $\left(1.2 \mu_{B}\right)$ but the obtained ordering temperature of $110 \mathrm{~K}$ is too low. Due to 5 closely lying discrete states the electronic structure and magnetism are very susceptible to lattice distortions and details of magnetic interactions. These five closely lying states are a physical reason for exotic experimental phenomena, when the $\mathrm{V}$ ion with such complex local structure interacts in a solid.

In conclusion, we have calculated the electronic structure of $\mathrm{V}_{2} \mathrm{O}_{3}$ associated with the $\mathrm{V}^{3+}$ ions taking into account strong on-site electron correlations and the spinorbit coupling. On basis of our studies we reject a possibility for an orbital liquid in $\mathrm{V}_{2} \mathrm{O}_{3}$. $\mathrm{V}^{3+}$ ions in the octahedral surroundings are strongly susceptible to lattice distortions and magnetic interactions. Our approach accounts both for the insulating ground state, magnetism as well as thermodynamical properties.

Answering a note of the SCES-05 first referee "While authors are right, that the single electron theory cannot fully describe many body effects, their schematic figure $1 \mathrm{~d}$ is wrong. The point is, that one does not speak about $E_{g}$ and $T_{2 g}$ "levels", but about $E_{g^{-}}$and $T_{2 g}$-like hybridizing "bands" of width few eV." we maintain that we do not believe that so wide bands exist in $\mathrm{V}_{2} \mathrm{O}_{3}$.

An extra remark. Despite the general critics of anonymous referees and discriminating policy of Editors of Phys. Rev. B and Phys. Rev. Lett., not mention others, we are convinced that such strongly-correlated CEF-based approach is the proper starting point for the physically adequate description of $3 d$ compounds. We call this approach Quantum Atomistic Solid State Theory (QUASST) - for more details about QUASST (cond-mat/0010081) and its application to a numerous compounds, e.g. $\mathrm{LaCoO}_{3}, \mathrm{CoO}, \mathrm{NiO}, \mathrm{FeBr}_{2}, \mathrm{LaMnO}_{3}$, one can find in our numerous papers in ArXiv and Z. Ropka, and R. J. Radwanski, Phys. Rev. B 67 (2003) 172401. It is obvious to remind that the free exchange of scientific information is essential for the development of Science.

- Dedicated to the Pope John Paul II, a man of freedom and truth in life and in Science.
[1] C. Castellani et al., Phys. Rev. B 18 (1978) 5001.

[2] S. Yu. Ezhov et al., Phys. Rev. Lett. 83 (1999) 4136.

[3] S. Di Matteo et al., Phys. Rev. 65 (2002) 054413.

[4] A. M. Oles, Acta Phys. Pol. B 32 (2001) 3303.

[5] P. Horsch et al., Phys. Rev. Lett. 91 (2003) 257203.

[6] A. Abragam and B. Bleaney, Electron Paramagnetic Res- onance of Transition Ions, Clarendon, Oxford, 1970.

[7] Y. Tanabe, S. Sugano, J. Phys. Soc. Japan 9 (1954) 753.

[8] Z. Ropka, R. Michalski, and R. J. Radwanski, Phys. Rev. B 63 (2001) 172404. 